\title{
Relative Weights for Pediatric Inpatients: Children Now Have a Scale of Their Own
}

\author{
Evan Fieldston, MD, MBA, MS*
}

Department of Pediatrics, Children's Hospital of Philadelphia and Perelman School of Medicine at the University of Pennsylvania, Philadelphia, Pennsylvania.

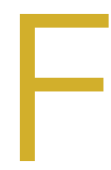

or the last 35 years, Medicare's prospective payment system has transformed reimbursement for hospital-based care of patients. This "revolutionary" system shifted payment from being retrospective - the government paid hospitals for what they did - to prospective the government paid hospitals against a predetermined fee schedule based on a patient's condition and other factors. ${ }^{1}$ When the system started in 1983, the then-new payment system classified patients into 467 Diagnosis-Related Groups (DRGs). In those early days, Medicare paid hospitals "an average price for an average patient within the DRG. ${ }^{2}$ Not surprisingly, early critics were concerned that this average payment would disadvantage hospitals that cared for more complex patients, such as teaching hospitals; studies then demonstrated that theoretical concern. ${ }^{3}$ The Severity of Illness (SOI) index, which was developed in the 1980s, attempted to correct this problem by using SOl-stratified DRGs as a payment mechanism. By adding SOI to DRGs, the homogeneity of resource consumption in each group increased, resulting in more accurate comparisons about complexity, outcomes, resource utilization, and ultimately payment. Eventually, along with the risk of mortality, the SOI made its way into the All Patients Refined (APR) DRG system, which is more representative of non-Medicare populations and thus could be applied to children.

The ongoing challenge with $\mathrm{SOI}$ classification is that its 4-level categories (1-mild, 2-moderate, 3-severe, 4-extreme) is not comparable across DRGs; that is, a "moderate" patient in one DRG may be sicker and use more resources than an "extreme" patient in another DRG. For this reason, more than a decade ago, Medicare replaced the DRG/SOI approach with the Medicare Severity (MS)-DRG for Medicare payments to hospitals. The distinguishing feature of MS-DRGs is that they represent a complete relative scale; the relative weights are not categorical but can be lined up and payments assigned relative to the average Medicare patient. For example, a look at the 2015 tables shows that heart transplant has the highest relative weight and is the most expensive one, whereas false labor has the lowest relative weight and is the least expensive. ${ }^{4}$ Due to its exclusive intent for use on Medicare patients, the

\footnotetext{
*Address for correspondence: Evan Fieldston, MD, MBA, MS, Department of Pediatrics, Children's Hospital of Philadelphia and Perelman School of Medicine at the University of Pennsylvania, Philadelphia, PA 19104; Telephone: 267-4262903; Fax: 267-426-6665; E-mail: fieldston@email.chop.edu

Published online first April 25, 2018.
}

Received: February 23, 2018; Accepted: March 3, 2017

๑) 2018 Society of Hospital Medicine DOI 10.12788/jhm.2982 system could not be used for pediatrics. Interestingly, New York State developed a Service Intensity Weight (SIW) in 2009 by using 3 years of Medicaid and commercial payer data to create a relative scale for payment within the state. ${ }^{5}$

Thanks to Richardson, et al, in this issue of Journal of Hospital Medicine, pediatrics has its first relative weight system for hospitalized children across the United States. ${ }^{6}$ Similar to the MS-DRG system, those with the interest or need can line up the APR-DRGs into a relative scale and see that a normal newborn has a relative weight on their H-RISK scale of 0.18 , while a heart transplant patient has a weight of 91.66. This is a welcome and much-needed addition to the world of pediatric health services and health service research. Stakeholders can use this system for comparative analyses, risk adjustment, resource utilization comparison, and payment. For those inclined, one can explore the comparisons of relative weights on different scales; for example, the ratio between simple pneumonia and heart transplant is 21 on the MS-DRG, 60 on the NY State SIW scale, and 187 on H-RISK. A generation of health service researchers and economists may find great satisfaction in elucidating why this relativity in relative scales exists!

There are limitations to all weighting and relative weighting systems. The H-RISK is based on DRG and SOI, which rely on accurate coding. In addition, as the authors note, iatrogenic complications are not differentiated from naturally occurring ones. Thus, a hospital may obtain a higher relative weight applied to a patient who did not enter the hospital as sick as the final score suggests. Researchers noted this problem from the start of the DRG/SOI journey, and all systems that rely on post hoc scoring based on coded diagnoses and activities, without differentiation of presence on admission, have this limitation. ${ }^{8}$ Furthermore, children's hospitals have far more variable use of observation status than in Medicare, and many DRG analyses exclude observation-status patients.

Despite these limitations, this is an important first step for children's hospitals to be better able to do comparative analyses and benchmarking with a true relative weight scale that is appropriate for use among hospitalized children.

Disclosure: The authors declare no conflicts of interest.

\section{References:}

1. Mayes R. The origins, development, and passage of Medicare's revolutionary prospective payment system. J Hist Med Allied Sci. 2007;62(1):21-55. DOI: 10.1093/jhmas/jij038.

2. Iglehart JK. Medicare begins prospective payment of hospitals. N Engl $J$ Med. 1983;303(23):1428-1432. DOI: 10.1056/NEJM198306093082331.

3. Horn SD, Sharkey PD, Chambers AF, Horn RA. Severity of illness within DRGs: 
impact on prospective payment. Am J Public Health. 1985;75(10):1195-1199. PMCID: PMC1646367

4. Inpatient Charge Data FY2015, https://www.cms.gov/Research-Statistics-Data-and-Systems/Statistics-Trends-and-Reports/Medicare-Provider-Charge-Data/Inpatient2015.html. Accessed February 20, 2018.

5. Service Intensity Weights (SIW) and average length-of-stay (LOS). https:// regs.health.ny.gov/content/section-86-118-service-intensity-weights-siwand-average-length-stay-los. Accessed February 22, 2018.

6. Richardson T, Rodean J, Harris M, Berry J, Gay JC, Hall M. Development of hospitalization resource intensity scores for kids (H-RISK) and comparison across pediatric populations. J Hosp Med. 2018;13(9):602-608. doi: 10.12788/ jhm.2948

7. APR-DRG Service Intensity Weights and Average Length of Stay, July 1, 2014. Department of Health, New York State. https://www.health.ny.gov/facilities/ hospital/reimbursement/apr-drg/weights/siw_alos_2014.htm. Accessed February 20, 2018.

8. Horn SD, Horn RA, Sharkey PD. The severity of illness index as a severity adjustment to diagnosis-related groups. Health Care Financ Rev. 1984;(Suppl):33-45. 